\title{
A New Pyrimidine-Specific Reporter Gene: A Mutated Human Deoxycytidine Kinase Suitable for PET During Treatment with Acycloguanosine-Based Cytotoxic Drugs
}

Yury Likar ${ }^{1}$, Juan Zurita ${ }^{1}$, Konstantin Dobrenkov ${ }^{1}$, Larissa Shenker ${ }^{1}$, Shangde Cai $^{1}$, Anton Neschadim², Jeffrey A. Medin ${ }^{2,3}$, Michel Sadelain ${ }^{4}$, Hedvig Hricak ${ }^{1}$, and Vladimir Ponomarev ${ }^{1}$

${ }^{I}$ Department of Radiology, Memorial Sloan-Kettering Cancer Center, New York, New York; ${ }^{2}$ Department of Medical Biophysics, University of Toronto, Toronto, Ontario, Canada; ${ }^{3}$ Ontario Cancer Institute, University Health Network, Toronto, Ontario, Canada; and ${ }^{4}$ Department of Medicine, Memorial Sloan-Kettering Cancer Center, New York, New York

\begin{abstract}
In this article, we describe a series of new human-derived reporter genes based on human deoxycytidine kinase (dCK) suitable for clinical PET. Methods: Native dCK and its mutant reporter genes were tested in vitro and in vivo for their phosphorylation of pyrimidine- and acycloguanosine-based radiotracers including 2'-deoxy-2'-fluoroarabinofuranosylcytosine, 2 '-fluoro-2'-deoxyarabinofuranosyl-5-ethyluracil (FEAU), penciclovir, and 9-[4-fluoro-3-(hydroxymethyl)butyl]guanine (FHBG) and clinically applied antiviral and anticancer drugs. Results: Cells transduced with dCK mutant reporter genes showed high in vitro and in vivo uptake of pyrimidine-based radiopharmaceuticals ( $\left.{ }^{18} \mathrm{~F}-\mathrm{FEAU}\right)$ comparable to that of herpes simplex virus type-1 thymidine kinase (HSV1-tk)-transduced cells. These mutants did not phosphorylate acycloguanosine-based radiotracers ( $\left.{ }^{18} \mathrm{~F}-\mathrm{FHBG}\right)$ or antiviral drugs (ganciclovir). Furthermore, the mutants displayed suicidal activation of clinically used pyrimidine-based prodrugs (cytarabine, gemcitabine). Conclusion: The mutants of human dCK can be used as pyrimidine-specific PET reporter genes for imaging with ${ }^{18} \mathrm{~F}-\mathrm{FEAU}$ during treatment with acycloguanosine-based antiviral drugs. Additionally, the prosuicidal activity of these reporters with pyrimidine-based analogs will allow for the safe elimination of transduced cells.

Key Words: molecular imaging; reporter gene; dCK; PET; FEAU; FHBG
\end{abstract}

J Nucl Med 2010; 51:1395-1403

DOI: 10.2967/jnumed.109.074344

\section{$\mathbf{R}$}

eportergene imaging technology represents a powerful tool for the study of different molecular-biologic processes in preclinical and clinical settings. The clinical application of PET-based reporter gene imaging for the noninvasive

Received Apr. 7, 2010; revision accepted May 11, 2010.

For correspondence or reprints contact: Vladimir Ponomarev, Molecular Imaging Laboratory, Department of Radiology, Memorial Sloan-Kettering Cancer Center, 1275 York Ave., Box 501, Z-2063, New York, NY 10021.

E-mail: ponomarv@mskcc.org

COPYRIGHT @ 2010 by the Society of Nuclear Medicine, Inc. monitoring of transduced cells in modern gene and cell therapies will expand over the next several years. Some reporter genes of different origins have been successfully tested in animal models as potential markers for clinical use (1). Herpes simplex virus type-1 thymidine kinase (HSV1-tk), one of the most commonly used reporter genes with PET, has been used in experimental models and clinical trials to evaluate responses to HSV1-tk-mediated suicide gene therapy (2-3), track the migration of HSV1-tk-expressing cells (4-7), and noninvasively assess the activity of endogenous gene expression (8-10).Despite the advantages of the HSV1-tk reporter gene- such as its high phosphorylation activity toward different pyrimidine-based ( 2 '-fluoro- 2 'deoxy-1 $\beta$-D-arabinofuranosyl-5-iodouracil [FIAU], $2^{\prime}$-fluoro-2'-deoxyarabinofuranosyl-5-ethyluracil [FEAU], 1-(2'deoxy-2'-fluoro- $\beta$-D-arabinofuranosyl)-5-(2-fluoroethyl) uridine, 1 - $\left(2^{\prime}\right.$-deoxy- $2^{\prime}$-fluoro- $\beta$-D-arabinofuranosyl $)-5$-bromouridine) and acycloguanosine-based (9-[4-fluoro-3-(hydroxymethyl)butyl]guanine [FHBG], 9-[3-fluoro-1-hydroxy2-propoxymethyl]guanine) radiotracers and its potent suicide function in activation of ganciclovir (Cytovene-IV; Roche Laboratories Inc.)—-several limitations could significantly devalue its use in clinical studies. For example, the rejection of HSV1-tk-expressing cells by an immunocompetent host suggests that strategies to render gene-modified cells less susceptible to host immune surveillance are required for successful gene therapy in this context (11-13). Furthermore, administration of ganciclovir, which is routinely used for antiviral therapy in immunocompromised patients who have received bone marrow (stem cell) transplants, will eliminate the adoptively transferred stem cells or lymphocytes transduced with the HSV1-tk reporter gene. To address this latter concern, we recently assessed a mutant of HSV1-tk bearing an arginine-to-glutamine substitution at position 176 that showed reduced phosphorylation activity toward acyclo- 
guanosine-based analogs while preserving pronounced enzymatic characteristics with pyrimidine derivatives (14).

To circumvent these limitations of HSV1-tk, we and others tested different endogenous and thus intrinsically nonimmunogenic reporter genes for potential use in patients. Among these were sodium iodide symporter (15), norepinephrine transporter (16), somatostatin receptor (17), dopamine receptor (18), ferritin (19), and transferrin receptor (20). A new human-derived reporter gene described by our group, human truncated mitochondrial thymidine kinase type 2 (h $\Delta \mathrm{TK} 2)(21)$, was shown to exclusively phosphorylate pyrimidine-based analogs, including ${ }^{124}$ I-FIAU and ${ }^{18} \mathrm{~F}-$ FEAU (albeit at moderate levels when compared with HSV1-tk).

This study focuses on another human nucleotide kinase, human deoxycytidine kinase (dCK), that is capable of converting deoxycytidine, deoxyadenosine, deoxyguanosine, and several pyrimidine-based cytotoxic drugs including cytosine-arabinoside (Ara-C [Cytarabine; Bedford Laboratories]) and 2'-difluoro-cytosine (gemcitabine [Gemzar; Eli Lilly and Co.) into their monophosphate forms (22). A new radiotracer, ${ }^{18} \mathrm{~F}-2$ '-deoxy-2'-fluoroarabinofuranosylcytosine $\left({ }^{18} \mathrm{~F}-\mathrm{FAC}\right)$, has been successfully used to visualize endogenous dCK activity in activated lymphoid tissue in vivo (23). It has been shown that select mutations at the active site of dCK improve enzyme kinetics and expand the catalytic repertoire of dCK to include thymidine (24-26). We hypothesize that a mutant of $\mathrm{dCK}$, bearing a thymidinepermissive conformation, could be investigated as a PET reporter gene in concert with thymidine-based radiotracers.

In this study, we evaluate the ability of human $\mathrm{dCK}$ and mutant forms to phosphorylate clinically applicable nucleoside-based radiotracers suitable for PET. We show that the dCK mutants phosphorylate pyrimidine-based radiotracers at levels comparable with that of wild-type HSV1-tk, PET of mutant dCK reporter gene expression using ${ }^{18} \mathrm{~F}$-FEAU is feasible, and mutant dCK-transduced cells are not sensitive to acycloguanosine analogs, whereas they are sensitive to pyrimidine-based prodrugs.

\section{MATERIALS AND METHODS}

\section{Oncoretroviral Vectors}

The amino acid sequences of human $\mathrm{dCK}$ and mutants used in this study are shown in Supplemental Table 1 (supplemental materials are available online only at http://jnm.snmjournals.org). Oncoretroviral vectors containing the complementary DNA for wild-type HSV1-tk and a mutant of HSV1-sr39tk bearing an arginine-to-glutamine substitution at position 176 (R176Q) with a nuclear export signal (NES) from mitogen-activated protein kinase kinase of Xenopus, SFG-NES-wild-type-HSV1-tk/green fluorescent protein (GFP) (HSV1-tk) and SFG-NES-HSV1-R176Qsr39tk/GFP (R176Qsr39tk), were described previously $(14,27)$ and served as reference vectors. Three complementary DNAs encoding native $\mathrm{dCK}$, a deoxycytidine kinase double mutant (dCKDM) containing arginine-to-methionine (position 104, R104 M) and aspartic acid-toalanine (positions 133, D133A) substitutions, and deoxycytidine kinase triple mutant (dCKTM) - a mutant of dCKDM bearing an additional serine-to-glutamic acid substitution at position 74 (S74E) (28)—were amplified using primers 5' - AGCTAGCTCCATGGCCACCCCGCCCAAG and 3'- AGCTAGCTGTTAACCAAAGTACTCAAAAACTC and introduced into the SFG-Neswild-type-HSV1-tk/GFP vector. These 3 complementary DNAs replaced the Nes-wild-type-HSV1-tk, which resulted in SFGdCK/GFP (dCK), SFG-dCKDM/GFP (dCKDM), and SFGdCKTM/GFP (dCKTM) oncoretroviral vectors. A mutant of dCKDM lacking nuclear import signal (nuclear localization signal, 5 first amino acids) in the $\mathrm{N}$ terminus replaced dCKDM in the SFG-dCKDM/GFP vector, resulting in an SFG- $\triangle \mathrm{dCKDM} / \mathrm{GFP}$ $(\triangle \mathrm{dCKDM})$ oncoretroviral vector. Oncoretroviral vectors encoding for human prostate-specific membrane antigen (hPSMA), hPSMA-directed chimeric antigen receptor Pz1, and B7.1 costimulatory ligand were described previously (29).

\section{Transduction of Tumor Cells}

The in vitro transduction of human glioma U87 cells with the recombinant oncoretroviral vectors was accomplished for $8 \mathrm{~h}$ in the presence of polybrene ( $8 \mu \mathrm{g} / \mathrm{mL}$; Sigma) as previously described (30). PC3 human prostate carcinoma cell line expressing hPSMA and NIH3T3 cells expressing B7.1 (CD80) and hPSMA were described previously (31). Fresh viral supernatants from PG13 cells (American Type Culture Collection) were used for T-cell transduction.

\section{T-Cell Transduction and Expansion}

Peripheral blood $(100 \mathrm{~mL})$ was drawn from healthy donors who had provided informed consent. Forty-eight hours after stimulation with phytohemagglutinin $(2 \mu \mathrm{g} / \mathrm{mL}) \mathrm{T}$ cells were transduced with Pz1-encoding retroviral vector as described previously (31) in the presence of IL-2 (20 units/mL). On day 7, Pz1-transduced T cells were selectively expanded by cocultivation with NIH3T3 target cells expressing B7.1 and hPSMA. Forty-eight hours after restimulation on target cells positive for NIH3T3/B7.1 and hPSMA, lymphocytes were transduced with SFG-dCKDMGFP or SFG-HSV1-tkGFP retroviral vectors as described previously (31). During the T-cell expansion, the medium was supplemented with interleukin-15 (10 ng/mL) every $3 \mathrm{~d}$.

\section{Flow Cytometry and Fluorescent Microscopy}

Oncoretrovirally transduced U87 cells were grown as bulk cultures for $48 \mathrm{~h}$ and subsequently sorted for uniform GFP expression using fluorescence-activated cell sorting (FACS) (FACSVantage; BD Biosciences). Subcellular localization of the reporter proteins in transduced tumor cells was visualized by fluorescence microscopy (Nikon) using similar excitation and emission parameters. Pz1 chimeric antigen receptor expression was detected using phycoerythrin-conjugated cross-reactive goat antimouse $\operatorname{IgG}$ (Calbiochem) and phycoerythrin-conjugated $\mathrm{F}$ $\left(\mathrm{ab}^{\prime}\right)_{2}$ antihuman IgG1 (Southern Biotechnology), respectively. hPSMAs were detected on tumor cells using J591 antibody, kindly provided by Dr. Neal Bander (Weill Medical College of Cornell University). Stained cells were processed on a FACScan and analyzed with Cell Quest software (BD Biosciences).

\section{Target-Specific Cell Lysis Assay}

The cytolytic activity of transduced T cells was assessed in 4-h aCella-TOX nonradioactive cytotoxicity assay (Cell Technology). Antigen-specific cytotoxicity was calculated according to the following formula: percentage of specific lysis $=$ (experimental release - spontaneous release)/(maximum release - spontaneous 
release) $\times 100$. Western blot analysis was performed as described previously (14). Proteins were detected using mouse monoclonal antibodies specific for human dCK (Abcam) and GFP (clone 7.1; Roche). Mouse $\beta$-actin-specific antibodies (Bio-Rad), alkaline phosphatase-conjugated goat antimouse antibody (Bio-Rad), and alkaline phosphatase-specific color development solution (Bio$\mathrm{Rad})$ were used for visualization. Chemiluminescence signals were collected on the Epi ChemiDoc imager (Bio-Rad) and quantified with QuantiOne Analysis software (Bio-Rad).

\section{${ }^{3} \mathrm{H}-\mathrm{FEAU},{ }^{14} \mathrm{C}-\mathrm{FIAU},{ }^{3} \mathrm{H}-$ Penciclovir, and ${ }^{3} \mathrm{H}-\mathrm{FAC}$ In Vitro Accumulation Assay}

The ${ }^{3} \mathrm{H}-\mathrm{FEAU},{ }^{14} \mathrm{C}-\mathrm{FIAU},{ }^{3} \mathrm{H}-$ penciclovir, and ${ }^{3} \mathrm{H}-\mathrm{FAC}$ accumulation assays were performed as previously described (32). Briefly, cells were seeded in $150 \times 25 \mathrm{~mm}$ tissue culture plates (Nunc) at a concentration of $2 \times 10^{6}$ cells per plate and grown until $50 \%-60 \%$ confluence. The incubation medium contained ${ }^{3} \mathrm{H}-$ FEAU $(3.7 \mathrm{kBq} / \mathrm{mL} ; 1.48 \mathrm{TBq} / \mathrm{mmol}),{ }^{3} \mathrm{H}$-penciclovir $(3.7 \mathrm{kBq} /$ $\mathrm{mL} ; 1.11 \mathrm{TBq} / \mathrm{mmol}),{ }^{3} \mathrm{H}-\mathrm{FAC}(3.7 \mathrm{kBq} / \mathrm{mL} ; 1.11 \mathrm{TBq} / \mathrm{mmol})$, or ${ }^{14} \mathrm{C}$-FIAU $(0.37 \mathrm{kBq} / \mathrm{mL} ; 2.22 \mathrm{GBq} / \mathrm{mmol}$ ) (Moravek Biochemicals) (purity $>99 \%$ ). The cells were harvested by scraping after various periods of incubation $(30,60$, and $120 \mathrm{~min})$ and centrifuged at $1,250 \mathrm{~g}$. The cell pellets were weighed, reconstituted in solubilization buffer (Soluene-350; PerkinElmer) and scintillation buffer (Insta-Fluor Plus; PerkinElmer), and assayed for radioactivity concentration using a TriCarb $1600 \beta$-spectrometer (Packard) with standard ${ }^{3} \mathrm{H}$-channel counting. The data were expressed as a harvested cell-to-medium concentration ratio: $(\mathrm{dpm} / \mathrm{g}$ of cells $) /\left(\mathrm{dpm} / \mathrm{mL}\right.$ of medium). The rates of accumulation $\left(\mathrm{K}_{\mathrm{i}}\right)$ for FEAU, FIAU, penciclovir, and FAC were determined from the slope of the cell-to-medium ratios versus incubation time plots and have units of tracer clearance from the medium $(\mathrm{mL}$ of medium $/ \mathrm{min} / \mathrm{g}$ of cells).

Because of the limited number of transduced primary lymphocytes, radiotracer accumulation experiments were performed in 6-well plates, and the data were expressed as a harvested cell-tomedium concentration ratio normalized by protein concentration (Thermo Scientific): (dpm/mg of protein)/(dpm/mL of medium). The radiotracer accumulation was determined from the value of the cell-to-medium ratios versus incubation time at $120 \mathrm{~min}$ and has units of tracer clearance from the medium ( $\mathrm{mL}$ of medium $/ \mathrm{mg}$ of protein).

\section{Prodrug Sensitivity Assays}

To determine the cytotoxic effect (inhibitory concentration of $\left.50 \%\left[\mathrm{IC}_{50}\right]\right)$ of ganciclovir, gemcitabine $\mathrm{HCl}$, and Ara-C on nontransduced and transduced U87 cells and primary human lymphocytes, a WST-1 (Roche) cell viability assay was performed $4 \mathrm{~d}$ after exposure to the drugs. Cells were plated into 96-well microtiter plates at an initial density of 4,000 (for U87 cells) or 90,000 (for primary lymphocytes) cells per well; ganciclovir, gemcitabine $\mathrm{HCl}$, or Ara-C were added to sets of 8 wells for each concentration tested. The drug concentrations ranged from $1 \mathrm{nM}$ to $10 \mathrm{mM}$ for ganciclovir and $0.1 \mathrm{nM}$ to $1 \mathrm{mM}$ for gemcitabine $\mathrm{HCl}$ and Ara-C.

\section{Experimental Groups of Animals}

All animal studies were performed under a protocol approved by the Memorial Sloan-Kettering Cancer Center Institutional Animal Care and Use Committee. The mice were anesthetized using a 2\%:98\% isoflurane:oxygen gas mixture. All animals were sacrificed using $\mathrm{CO}_{2}$ inhalation. For subcutaneous xenograft experiments, 6- to 8 -wk-old nude mice from Taconic were used, and $5 \times$ $10^{6}$ U87 cells per tumor were implanted. Three groups of animals ( $n=10$ /group; 30 animals total) were studied, and 2 xenografts were produced in each animal. U87/dCKDM xenografts were established in the right shoulder of each animal in groups 1 and 2 . The second xenograft was established in the left shoulder from U87/nontransduced (group 1) and U87/dCK (group 2) cells. In the third group, xenografts were established from HSV1-tk- and R176Qsr39tktransduced cells in the left and right shoulders, respectively.

For the T-cell tracking experiments, immunodeficient 6- to 8 -wk-old severe combined immune-deficient/beige mice (Taconic) were used. To establish a lung metastatic tumor model of prostate cancer, mice were injected with $4 \times 10^{6}$ PC3/hPSMA-positive prostate carcinoma cells using tail vein injection. Pz1-transduced T-lymphocytes specific for hPSMA were cotransduced with the SFG-dCKDMGFP reporter vector. Pz1-positive/dCKDMGFPpositive double-transduced lymphocytes were adoptively transferred into PC3/hPSMA-positive tumor-bearing mice $\left(20 \times 10^{6} \%\right.$ animal) by tail vein injection. Nontreated tumor-bearing mice were used as a negative control.

\section{Micro-CT}

Registered CT images were acquired using a CT scanner (Gamma-Medica). The CT subsystem uses a complementary metal-oxide semiconductor detector and fixed tungsten anode radiographic source operating at a maximum voltage of $55 \mathrm{kVp}$ and anode current of approximately $0.60 \mathrm{~mA}$ and having a focal spot of approximately 50. Projection data were acquired in a cone-beam geometry with 360 approximately 1 -s steps at $1^{\circ}$ angular increments. Images were reconstructed using a 3-dimensional cone-beam (Feldkamp) filtered backprojection algorithm. Heart and blood vessel contrast was achieved by the intravenous administration of iodinated lipids (Fenestra VC; Alerion Biomedical Inc.).

\section{PET with ${ }^{18} \mathrm{~F}-\mathrm{FHBG}$ and ${ }^{18} \mathrm{~F}-\mathrm{FEAU}$}

The nude mice were monitored daily for tumor growth. Imaging studies were performed when subcutaneous tumors reached approximately $10 \mathrm{~mm}$ in diameter. ${ }^{18} \mathrm{~F}$-FHBG and ${ }^{18} \mathrm{~F}$-FEAU were prepared as previously described (33-34). Small-animal PET was performed at $2 \mathrm{~h}$ after tail vein administration of $7.4 \mathrm{MBq}(200$ $\mu \mathrm{Ci}$ ) of ${ }^{18} \mathrm{~F}-\mathrm{FHBG}$ (specific activity, $44.4 \mathrm{TBq} / \mathrm{mmol}$ ) to each animal. After the ${ }^{18} \mathrm{~F}$ radioactivity decay ( $24 \mathrm{~h}$ later), the same animals were injected via the tail vein with $7.4 \mathrm{MBq}(200 \mu \mathrm{Ci})$ of ${ }^{18}$ F-FEAU (specific activity, $37 \mathrm{TBq} / \mathrm{mmol}$ ) and imaged $2 \mathrm{~h}$ later. For T-cell tracking, 1 session of ${ }^{18}$ F-FEAU $(7.4 \mathrm{MBq}[200 \mu \mathrm{Ci}])$ small-animal PET was performed at $6 \mathrm{~h}$ after tail vein administration of T cells. PET was performed using a microPET Focus 120 scanner (Siemens Preclinical Solutions). At least 10 million coincidence events were acquired per study using a 350- to 750$\mathrm{keV}$ energy window and a 6-ns timing window. List-mode data were sorted into sinograms by Fourier rebinning and reconstructed by filtered backprojection without attenuation or scatter correction. Count data in the reconstructed images were converted to activity concentration (i.e., percentage of the injected dose per $\mathrm{cm}^{3}$ ) based on a system-calibration factor determined using an ${ }^{18} \mathrm{~F}$-filled mouse-sized phantom. Visualization and analyses of small-animal PET/micro-CT images were performed using AsiPRO software (Siemens Preclinical Solutions). Radioactivity concentration in tissue was calculated from the small-animal PET images using maximum pixel values. 
Tissue Sampling and Radioactivity Measurements

Immediately after the ${ }^{18} \mathrm{~F}$-FEAU and ${ }^{18} \mathrm{~F}$-FHBG imaging sessions, selected animals were sacrificed. Tumors and muscle tissue were excised, washed, and weighed. The radioactivity concentration in tissue samples was measured for 1 min using a $\gamma$-counter (model A5550; Packard), normalized to sample weight, and expressed as the percentage of injected dose per gram of tissue.

\section{Histology}

Histopathologic examination of the specimens was performed as described previously (35). For immunofluorescence analysis, freshly isolated tumor tissue was frozen in optimal-cutting-temperature compound (Sakura Finetek). The specimens were incubated with mouse anti-hPSMA monoclonal antibodies 7C12 (kindly provided by Dr. Polly Gregor, Memorial Sloan-Kettering Cancer Center) and anti-GFP rabbit polyclonal IgG (catalog no. A11122; Invitrogen), followed by secondary antibody antimouse Alexa Fluor 555 or antirabbit 488 (Invitrogen). For the visualization of the nuclei, 4,6-diamidino-2-phenylindole was used. Immunofluorescence was assessed using a fluorescence microscope (Axioplan-2; Carl Zeiss Microimaging Inc.).

\section{Statistical Analysis}

All cell culture and mouse group comparisons were performed with a Student $t$ test for independent samples with unequal variances. Mean values and the independent $t$ test for unequal variances were calculated using Prism 4 (GraphPad Software). $P$ values of less than 0.05 were considered statistically significant.

\section{RESULTS}

\section{Characterization of Reporter Genes In Vitro}

The subcellular localization of reporter gene products was assessed in transduced U87 cells by fluorescence microscopy, which demonstrated nuclear localization of $\mathrm{dCK} / \mathrm{GFP}$, dCKDM/GFP, and dCKTM/GFP reporter proteins. Cytoplasmic distribution of $\triangle \mathrm{dCKDM} / \mathrm{GFP}$ was observed, likely because of the deletion of the 5 amino acids containing a nuclear import signal in the $\mathrm{N}$ terminus (Fig. 1A). Transduced cells were sorted for GFP-positive populations with similar levels of GFP expression $(\sim 600$ fluorescent units). After FACS sorting, all cell lines were more than $95 \%$ GFP-positive.

The endogenous $\mathrm{dCK}$ and retrovirally expressed dCK/GFP, $\mathrm{dCKDM} / \mathrm{GFP}, \mathrm{dCKTM} / \mathrm{GFP}$, and $\triangle \mathrm{dCKDM} / \mathrm{GFP}$ fusion proteins had the predicted molecular mass of approximately 31 and approximately $59 \mathrm{kDa}$, respectively, as determined by Western blot analysis (Fig. 1B). The HSV1-tk/GFP fusion protein had a molecular mass of approximately $72 \mathrm{kDa}$.

The levels of radiotracer accumulation in vitro in native dCK, dCKDM, dCKTM, $\triangle \mathrm{dCKDM}$, HSV1-tk, and R176Qsr39tk-transduced U87 cells were assessed using a previously established assay with ${ }^{3} \mathrm{H}$-FEAU, ${ }^{3} \mathrm{H}$-penciclovir, and ${ }^{3} \mathrm{H}-\mathrm{FAC}$ substrates. Relatively high levels of ${ }^{3} \mathrm{H}-$ FAC accumulation were observed in nontransduced and HSV1-tk-transduced cells $\left(\mathrm{K}_{\mathrm{i}}, 0.66 \pm 0.28\right.$ and $0.87 \pm$ $0.18 \mathrm{~mL} / \mathrm{min} / \mathrm{g}$ of cells, respectively). Even higher ${ }^{3} \mathrm{H}-$ FAC uptake was obtained in cells transduced with native $\mathrm{dCK}, \mathrm{dCKDM}, \mathrm{dCKTM}$, and $\triangle \mathrm{dCKDM}\left(\mathrm{K}_{\mathrm{i}}, 2.88 \pm 0.90\right.$,
$5.72 \pm 0.42,5.97 \pm 0.51$, and $4.9 \pm 0.28 \mathrm{~mL} / \mathrm{min} / \mathrm{g}$ of cells, respectively; Fig. 1C). Nontransduced U87 cells had background levels of ${ }^{3} \mathrm{H}$-FEAU and ${ }^{3} \mathrm{H}$-penciclovir uptake $\left(\mathrm{K}_{\mathrm{i}}, 0.003 \mathrm{~mL} / \mathrm{min} / \mathrm{g}\right.$ of cells, Fig. 2). All cells transduced with native dCK or its mutants showed no ${ }^{3} \mathrm{H}$-penciclovir uptake, similar to nontransduced U87 cells, whereas ${ }^{3} \mathrm{H}-$ FEAU was efficiently accumulated by dCKDM-positive, dCKTM-positive, and $\triangle \mathrm{dCKDM}$-positive cells $\left(\mathrm{K}_{\mathrm{i}}, 0.32\right.$, 0.28 , and $0.35 \mathrm{~mL} / \mathrm{min} / \mathrm{g}$ of cells, respectively). In contrast, no ${ }^{3} \mathrm{H}$-FEAU accumulation was observed by native $\mathrm{dCK}$ cells. Cells transduced with HSV1-tk and R176Qsr39tk reporter genes showed high ${ }^{3} \mathrm{H}-\mathrm{FEAU}$ and moderate ${ }^{3} \mathrm{H}-$ penciclovir uptake (HSV1-tk $>$ R176Qsr39tk). ${ }^{14} \mathrm{C}$-FIAU accumulation paralleled ${ }^{3} \mathrm{H}-\mathrm{FEAU}$ uptake.

To explore the possibility of eliminating cells transduced with dCK-based reporter genes using a prodrug activation suicide approach, nontransduced and transduced U87 cells were tested for in vitro sensitivity to clinically relevant nucleoside analogs, including ganciclovir, gemcitabine, and Ara-C. Cells transduced with native dCK and its mutants were sensitive to Ara-C and gemcitabine. Only cells expressing HSV1-tk were exclusively sensitive to ganciclovir treatment (Supplemental Table 2).

\section{In Vivo Imaging of Reporter Gene Expression in Subcutaneous Xenografts with PET}

Small-animal PET studies were performed in mice bearing subcutaneous xenografts derived from transduced U87 cell populations and wild-type U87 cells (Fig. 3). The levels of ${ }^{18} \mathrm{~F}-\mathrm{FHBG}$ and ${ }^{18} \mathrm{~F}-\mathrm{FEAU}$ radioactivity in control (nontransduced) U87 tumors were low, similar to the body's background levels. ${ }^{18} \mathrm{~F}-\mathrm{FHBG}$ accumulation was observed neither in nontransduced xenografts nor in native $\mathrm{dCK}$ and dCKDM-transduced tumors. Detectable levels of ${ }^{18} \mathrm{~F}$ FHBG accumulation were measured in HSV1-tk-positive and R176Qsr39tk-positive tumors. Small-animal PET revealed a high accumulation of ${ }^{18} \mathrm{~F}-\mathrm{FEAU}$ in xenografts expressing dCKDM, HSV1-tk, and R176Qsr39tk reporter genes, with a marginal advantage favoring the HSV1-tk (Fig. 3). No ${ }^{18}$ F-FEAU accumulation was observed in xenografts expressing native $\mathrm{dCK}$ and in nontransduced tumors.

Tissue sampling and biodistribution studies confirmed highly specific ${ }^{18}$ F-FEAU accumulation in dCKDM-positive, HSV1-tk-positive, and R176Qsr39tk-positive tumors, whereas ${ }^{18} \mathrm{~F}-\mathrm{FHBG}$ accumulation was observed in HSV1tk-positive and R176Qsr39tk-positive xenografts (Supplemental Fig. 1). The tumors transduced with native dCK, nontransduced U87 tumors, and muscle tissue showed only background levels of ${ }^{18} \mathrm{~F}$-FEAU radioactivity.

\section{Characterization of dCKDM-Transduced T Cells In Vitro}

Cell-surface expression of anti-PSMA chimeric antigen receptor Pz1 and GFP fluorescence were assessed by FACS. Pz1-positive T cells were $75 \%$ and $78 \%$ double-positive for dCKDM/GFP-positive and HSV1tk/GFP, respectively. Cotransduction of $\mathrm{T}$ cells with dCKDM/GFP and HSV1tk/ 


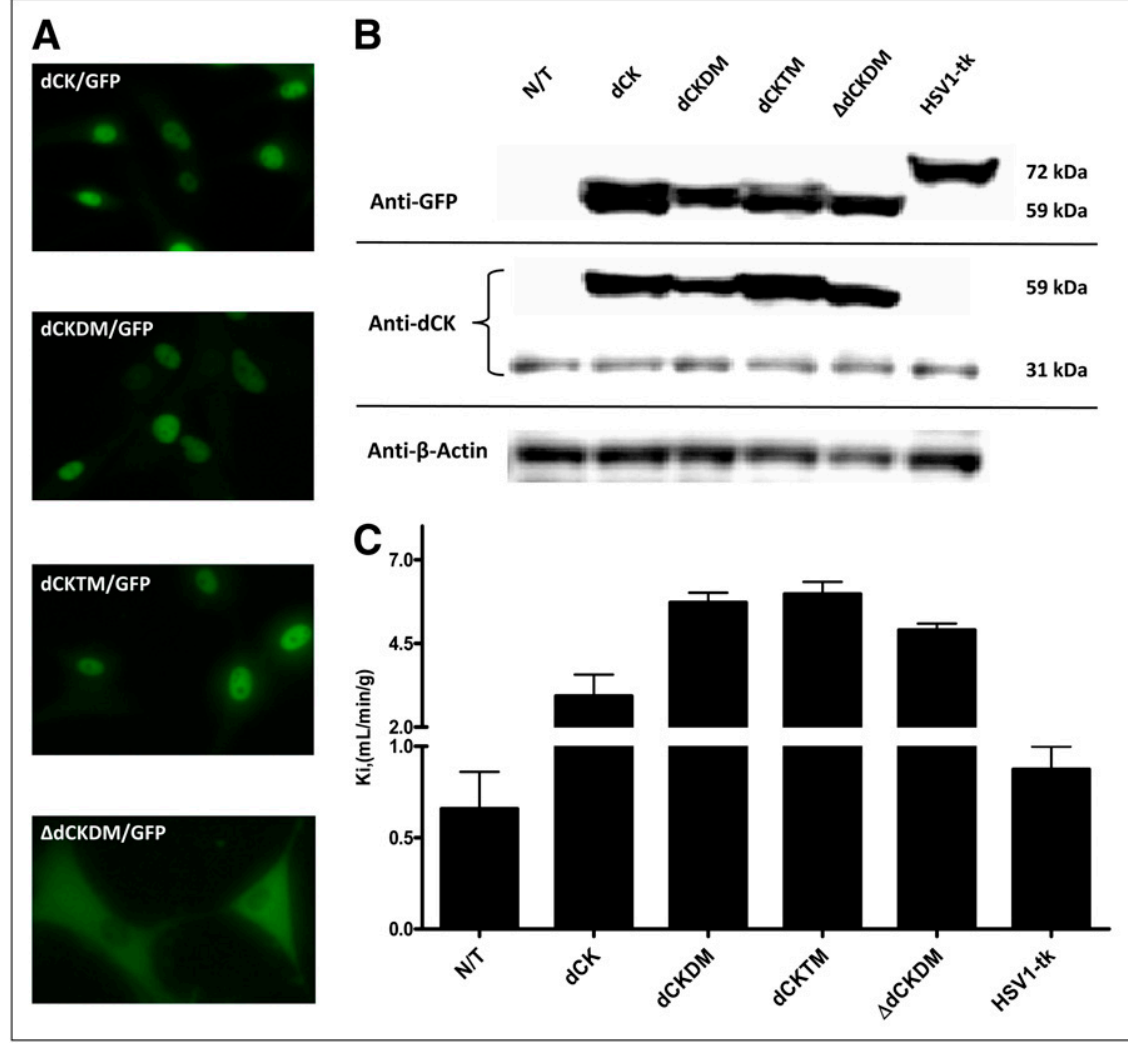

FIGURE 1. Assessment of reporter gene expression in vitro. (A) Subcellular localization of native-dCK/GFP, dCKDM/GFP, dCKTM/GFP, and $\triangle \mathrm{dCK} /$ GFP reporter proteins. (B) Western blot analysis of $\mathrm{dCK}$ and mutant protein expression in nontransduced and transduced cell populations using human dCK and GFP- and $\beta$-actin-specific monoclonal antibodies. (C) ${ }^{3} \mathrm{H}-\mathrm{FAC}$ uptake $\left(\mathrm{K}_{\mathrm{i}}\right)$ in nontransduced and transduced U87 cells is listed on abscissa. Values are mean $\pm \mathrm{SD}, n=3 . \mathrm{N} / \mathrm{T}=$ nontransduced.
GFP reporter genes did not affect their cytolytic activity against PC3/PSMA-positive target cells. Nontransduced and $\mathrm{dCKDM} / \mathrm{GFP}$-expressing $\mathrm{T}$ cells were not sensitive to ganciclovir treatment $\left(\mathrm{IC}_{50}>500 \mu \mathrm{M}\right)$, whereas HSV1-tk/ GFP-positive lymphocytes showed increased sensitivity toward ganciclovir $\left(\mathrm{IC}_{50}, 7 \mu \mathrm{M}\right) .{ }^{3} \mathrm{H}-\mathrm{FEAU}$ accumulation in HSV1-tk/GFP- and dCKDM/GFP-transduced lymphocytes in vitro was significantly higher than that in nontransduced cells. The data are summarized in Supplemental Fig. 2.

\section{PET/CT of dCKDM-Transduced Lymphocytes}

$\mathrm{T}$ cells transduced with Pz1 and dCKDM/GFP reporter gene were administered by tail vein injection into the mice with pulmonary PC3/hPSMA-positive tumors. By that time, extensive tumors were detectable by micro-CT in all animals (Fig. 4). A separate group of tumor-bearing animals was used as a negative control. Small-animal PET/micro-CT of dCKDM-expressing $\mathrm{T}$ cells targeting PSMA-positive tumors was performed on the day of T-cell injection. Distinct ${ }^{18} \mathrm{~F}$-FEAU accumulation in the lung tumors in animals injected with dCKDM-transduced T cells (Fig. 4A), compared with nontreated animals (Fig. 4B), was observed. The focus-to-background ratios at the tumor site were significantly higher in this group than in the nontreated group $(3.06 \pm 0.9$ vs. $1.06 \pm 0.07, P<0.05$, Figs. $4 \mathrm{E}$ and $4 \mathrm{~F})$.

The presence of tumors infiltrating normal-lung parenchyma was shown using immunofluorescent staining for
hPSMA in both groups (Figs. 4C and 4D). Tumor specimens taken from animals treated with dCKDM/GFPpositive $\mathrm{T}$ cells contained GFP-positive cells, whereas no GFP-positive cells were found in specimens taken from nontreated animals. In addition, fluorescent microscopy revealed localization of GFP-positive cells surrounding PSMA-positive tumors in the animals treated with dCKDM/GFP-expressing T cells.

\section{DISCUSSION}

Being intrinsically nonimmunogenic, human-derived reporter genes will play an increasingly important role in noninvasive monitoring of the efficacy of gene and cellular therapies in humans. It is anticipated that one or more human reporter systems (human-derived reporter gene and complementary probe) will take a leading role in upcoming clinical studies of cancer treatment and other debilitating diseases (e.g., metabolic disorders or inherited genetic deficiencies). Native and genetically engineered human deoxyribonucleoside kinases are attractive candidates for use in gene and cell therapies as suicide enzymes and reporter genes. These reporters will most likely be nonimmunogenic in patients. However, only future clinical studies will allow the assessment of human reporter genes in a physiologic context.

Our group described a new human-derived reporter gene, truncated $\mathrm{h} \Delta \mathrm{TK} 2$, which is intrinsically restricted to 


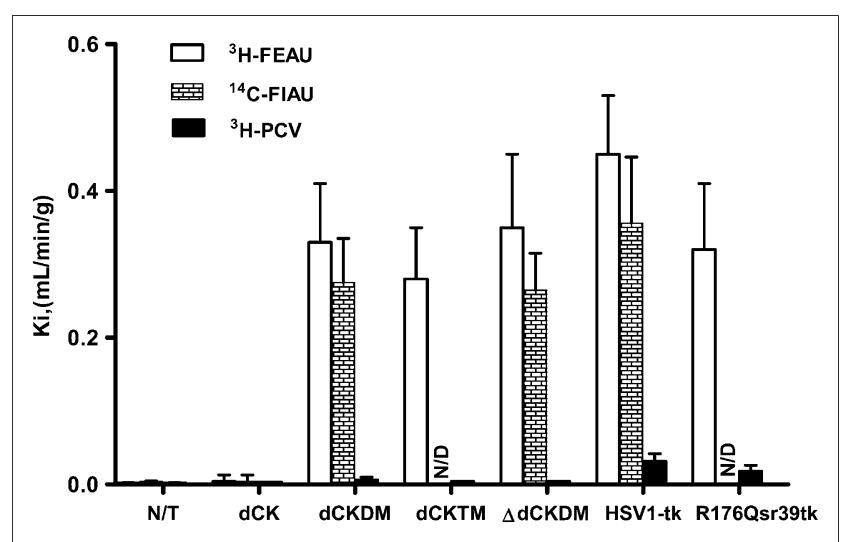

FIGURE 2. Radiotracer accumulation in transduced cells in vitro. Radiotracer uptake $\left(\mathrm{K}_{\mathrm{i}}\right)$ of ${ }^{3} \mathrm{H}-\mathrm{FEAU},{ }^{14} \mathrm{C}-\mathrm{FIAU}$, and ${ }^{3} \mathrm{H}-$ penciclovir in nontransduced and transduced U87 cells is listed on abscissa. Values are mean $\pm \mathrm{SD}, n=3, P<$ 0.05. N/D = not done; $\mathrm{N} / \mathrm{T}=$ nontransduced; $\mathrm{PCV}=$ penciclovir.

pyrimidine-based nucleosides (21). We have demonstrated that cells transduced with $\mathrm{h} \Delta \mathrm{TK} 2$ do not accumulate acycloguanosine derivatives such as ${ }^{3} \mathrm{H}$-penciclovir and ${ }^{18} \mathrm{~F}-\mathrm{FHBG}$ and can be imaged with pyrimidine-based radiotracers including ${ }^{124}$ I-FIAU and ${ }^{18}$ F-FEAU. However, cells expressing $\mathrm{h} \Delta \mathrm{TK} 2$ showed relatively low levels of pyrimidine-based radiotracer accumulation (6- to 10-fold lower than those of HSV1-tk-positive cells). This limitation of $\mathrm{h} \Delta \mathrm{TK} 2$ prompted us to search for a more active human nucleoside-based reporter gene candidate.

Our subsequent studies focused on human dCK, an essential enzyme for the phosphorylation of numerous pyrimidine-based prodrugs routinely used in cancer and antiviral chemotherapy including Ara-C, gemcitabine, fluorodeoxycytidine, $2^{\prime}$-fluoro-arabinocytosine, and the like (22). We and others (23) have demonstrated that ${ }^{18} \mathrm{~F}-\mathrm{FAC}$ is a good radiotracer for imaging endogenous dCK activity. However, only a moderate increase of ${ }^{18} \mathrm{~F}-\mathrm{FAC}$ radiotracer accumulation ( $\sim 3$-fold, Fig. 1C) was achieved in native dCK-transduced cells, compared with wild-type cells. Several groups have studied mutants of dCK to improve enzyme kinetics for suicide gene therapy applications and expand the catalytic repertoire of dCK $(24-26,28)$. In this study, we demonstrated that the cells expressing $\mathrm{dCKDM}$ bearing R104 M and D133A substitutions, when compared with native $\mathrm{dCK}$, have an increased rate of accumulation of radiolabeled thymidine analogs. We have explored the $\mathrm{dCKDM}$ mutant as a PET reporter gene in combination with clinically applicable pyrimidine-based radiotracers. Our in vitro radiotracer accumulation and in vivo imaging studies demonstrated the advantage of this mutant reporter gene over its native predecessor in phosphorylating FEAU/ FIAU because of a thymidine-permissive conformation of this enzyme (dCKDM/dCK-transduced ratio of $\sim 100)$. Furthermore, both PET and direct measurement of radiotracer
FIGURE 3. Small-animal PET of reporter gene expression. (A) Coronal small-animal PET images through xenografts placed subcutaneously over shoulders are shown: nontransduced and transduced U87 xenografts, including dCK, dCKDM, HSV1-tk, and HSV1R176Qsr39tk. ${ }^{18} \mathrm{~F}-\mathrm{FEAU}$ and ${ }^{18} \mathrm{~F}-\mathrm{FHBG}$ images at $2 \mathrm{~h}$ after radiotracer administration obtained on consecutive days are shown for same animal. All images were adjusted to same color scale. (B) Image-based measurements of ${ }^{18} \mathrm{~F}$ FEAU and ${ }^{18} \mathrm{~F}-\mathrm{FHBG}$ at $2 \mathrm{~h}$ after radiotracer administration in same animals, expressed as percentage injected dose per cubic centimeter of tissue (\%ID/ $\left.\mathrm{cm}^{3}\right)$. Values are mean $\pm \mathrm{SD}, n=5$. $\mathrm{N} / \mathrm{T}=$ nontransduced.

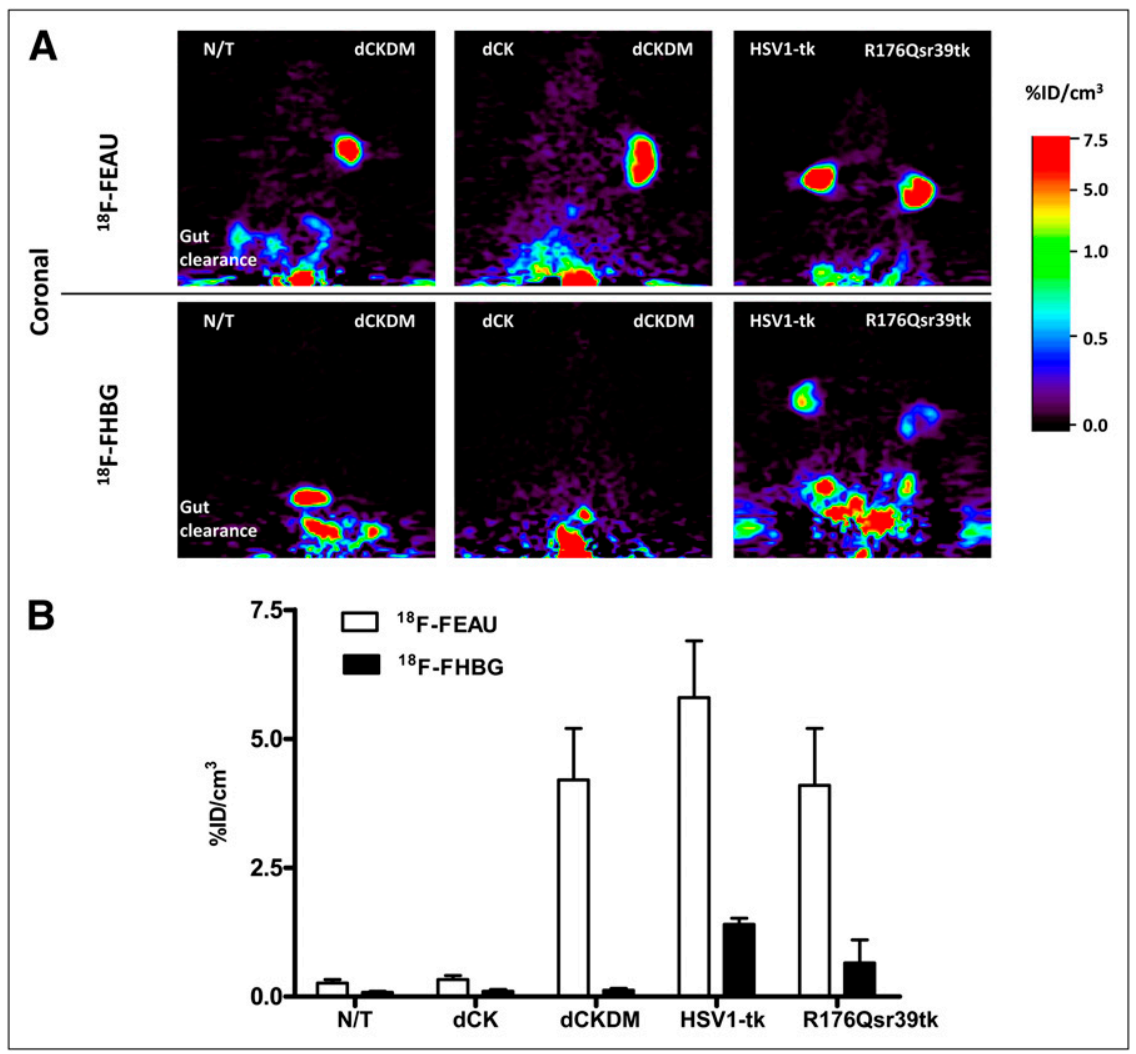




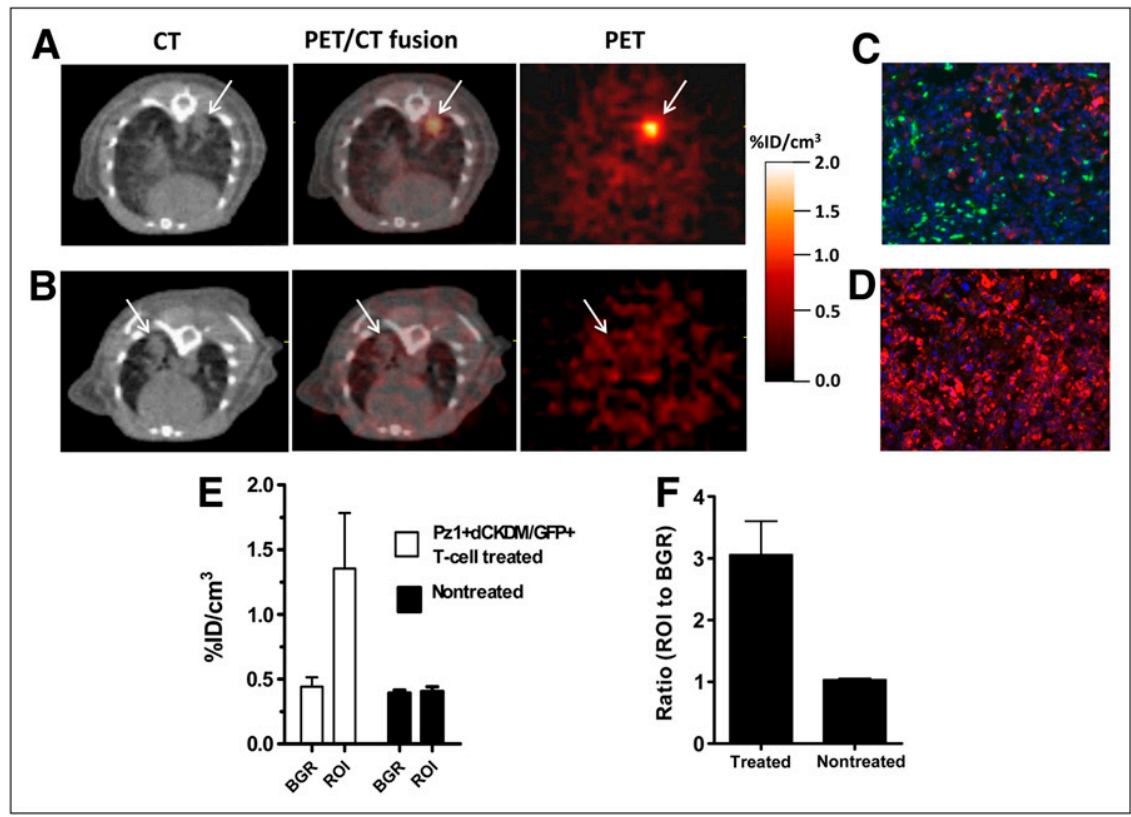

FIGURE 4. Small-animal PET/microCT of dCKDM/GFP-expressing T cells: colocalization of ${ }^{18} \mathrm{~F}-\mathrm{FEAU}$ accumulation in small-animal PET images with tumor regions in micro-CT images. Representative tumor-bearing animals from $P z 1+d C K D M / G F P+$ treated $(A)$ and control nontreated (B) groups are shown. High ${ }^{18}$ F-FEAU accumulation was detected on day of T-cell administration in region corresponding to tumor foci on micro-CT image in Pz1+dCKDM/GFP+ treated animal. No ${ }^{18} \mathrm{~F}-\mathrm{FEAU}$ accumulation was observed in region corresponding to tumor foci in nontreated control animal. Heart and blood vessels were enhanced by iodinated lipids. Immunofluorescent analysis revealed presence of GFP-positive (green, stained with anti-GFP antibody) $T$ cells infiltrating hPSMA-positive (red, stained with anti-hPSMA antibody) tumor in lung from representative Pz1+dCKDM/GFP+ T-cell-treated (C) and -nontreated tumor-bearing (D) animals. Nuclei were stained with 4,6-diamidino-2-phenylindole (blue). Magnification, $\times 10$. Quantitative analysis of ${ }^{18} \mathrm{~F}-\mathrm{FEAU}$-accumulation $(\mathrm{E})$ and region-of-interest-to-background ratios $(\mathrm{F})$ in treated and nontreated groups is presented. Data are expressed as percentage injected dose per cubic centimeter of tissue $\left(\% \mathrm{ID} / \mathrm{cm}^{3}\right)$. Values are mean $\pm \mathrm{SD}, n=5$. BGR = background; $\mathrm{ROI}=$ region of interest, marked with arrow.

accumulation in tissue samples confirmed high ${ }^{18} \mathrm{~F}-\mathrm{FEAU}$ uptake in dCKDM-expressing tumors, comparable with the uptake of HSV1-tk-positive and R176Qsr39tk-positive xenografts. The latter result makes this mutant of dCK an attractive candidate for preclinical and clinical PET of transduced cells and tissues using arabinouracil-based radiotracers labeled with short-lived radioisotopes.

A reporter gene should not alter the phenotype and function of the transduced cells. Here in this study, we showed that the expression of the mutant dCK-based reporter in primary human $\mathrm{T}$ lymphocytes did not affect their cytolytic activity against target cells and allowed successful PET of systemically administered $\mathrm{T}$ cells targeting tumors in a previously described prostate metastatic cancer model $(5,31,36)$. This imaging paradigm could be incorporated into clinical protocols of adoptive immunotherapy for the monitoring of T-cell trafficking, persistence, and early prediction of therapy outcome.

For over 2 decades, acycloguanosine-based drugs have been routinely used for antiviral therapy in immunocompromised patients undergoing immunosuppressive regimens for anticancer treatment and bone marrow (stem cell) transplantation (e.g., ganciclovir for cytomegalovirus infection or acyclovir for herpes simplex infection) (3738). The antiviral administration of ganciclovir or acyclovir in patients undergoing gene or cell therapy will be detrimental to cells transduced with HSV1-tk-based reporters and to the negative selection of HSV1-tk-expressing cells that will adversely impact HSV1-tk reporter imaging. In our experiments, dCKDM-expressing cells including primary human lymphocytes were not as sensitive to ganciclovir treatment as the nontransduced cells. These data suggest that the dCKDM reporter gene- ${ }^{18}$ F-FEAU reporter probe system can be successfully used in patients treated with acycloguanosine-based drugs, without altering the viability of reporter gene-expressing cells.

Ideally, a clinically used reporter gene could also be expected to work as a safety switch for the successful elimination of genetically modified cells when needed. Our and previously published data (28) showed the increased sensitivity of mutant dCK-transduced cells, when compared with the sensitivity of nontransduced cells, to Ara$\mathrm{C}$ and gemcitabine (10-fold). The finding suggests that the dCKDM reporter can be potentially used for suicide prodrug activation of clinically relevant nucleoside analogs gemcitabine and Ara-C for the selective removal of transduced cells in vivo.

In this study, we evaluated 2 additional mutants of the dCKDM reporter gene. According to McSorley et al. (39), the S74E mutation in native dCK increases the catalytic constant values 11-fold for deoxycytidine and 3-fold for Ara-C. In our studies, little discernable difference between the kinase activities of the dCKDM and dCKTM reporter proteins with FEAU was observed. In addition, we tested a mutant of dCKDM with truncated nuclear localization signal at the $\mathrm{N}$ terminus (40). This deletion resulted in pancellular distribution of $\triangle \mathrm{dCKDM}$ reporter protein, whereas the $\triangle \mathrm{dCKDM}$-transduced cells showed an insignificant 
increase in ${ }^{3} \mathrm{H}-\mathrm{FEAU}$ accumulation in vitro due to improved reporter protein availability in the cytoplasm of transduced cells. Therefore, the $\triangle \mathrm{dCKDM}$ reporter gene can be successfully used in studies that require cytoplasmic distribution of the reporter protein (e.g., coexpression with other nucleuslocalized fluorescent proteins or concurrent immunofluorescent analysis of nuclear protein distribution).

\section{CONCLUSION}

We have successfully tested in vitro and in vivo a new mutant dCKDM human reporter gene with expanded thymidine kinase activity. This reporter lacks the ability to convert acycloguanosine-based nucleoside derivatives but exhibits high phosphorylation activity with thymidine analogs. This mutant can be used as a pyrimidine-specific PET reporter gene for imaging with ${ }^{18}$ F-FEAU in patients treated with acycloguanosine-based antiviral drugs and as a suicide gene for prodrug activation of clinically relevant pyrimidine analogs.

\section{ACKNOWLEDGMENTS}

We thank Dr. Pat Zanzonico and Laurence James for their technical assistance and expertise. We thank Dr. Ronald Blasberg for his help in preparing this manuscript. We also thank Alex Hantman and Elias Kleinbock for their technical assistance in editing this manuscript. This work was supported by NIH grants P50 CA86438-01 and R01 CA102352, NIH Small-Animal Imaging Research Program (SAIRP) grant R24 CA83084, NIH Center grant P30 CA08748, the William H. Goodwin and Alice Goodwin Commonwealth Foundation for Cancer Research, and the Experimental Therapeutics Center of Memorial SloanKettering Cancer Center. Technical services were provided by the MSKCC Small-Animal Imaging Core Facility.

\section{REFERENCES}

1. Min JJ, Gambhir SS. Molecular imaging of PET reporter gene expression. Handb Exp Pharmacol. 2008;185/2:277-303.

2. Haberkorn U, Altmann A, Morr I, et al. Monitoring gene therapy with herpes simplex virus thymidine kinase in hepatoma cells: uptake of specific substrates. J Nucl Med. 1997;38:287-294.

3. Yaghoubi SS, Barrio JR, Namavari M, et al. Imaging progress of herpes simplex virus type 1 thymidine kinase suicide gene therapy in living subjects with positron emission tomography. Cancer Gene Ther. 2005;12:329-339.

4. Koehne G, Doubrovin M, Doubrovina E, et al. Serial in vivo imaging of the targeted migration of human HSV-TK-transduced antigen-specific lymphocytes. Nat Biotechnol. 2003;21:405-413.

5. Dobrenkov K, Olszewska M, Likar Y, et al. Monitoring the efficacy of adoptively transferred prostate cancer-targeted human $\mathrm{T}$ lymphocytes with PET and bioluminescence imaging. J Nucl Med. 2008;49:1162-1170.

6. Dubey P, Su H, Adonai N, et al. Quantitative imaging of the T cell antitumor response by positron-emission tomography. Proc Natl Acad Sci USA. 2003;100:1232-1237.

7. Yaghoubi SS, Jensen MC, Satyamurthy N, et al. Noninvasive detection of therapeutic cytolytic T cells with ${ }^{18} \mathrm{~F}$-FHBG PET in a patient with glioma. Nat Clin Pract Oncol. 2009;6:53-58.

8. Doubrovin M, Ponomarev V, Beresten T, et al. Imaging transcriptional regulation of p53-dependent genes with positron emission tomography in vivo. Proc Natl Acad Sci USA. 2001;98:9300-9305.
9. Ponomarev V, Doubrovin M, Lyddane C, et al. Imaging TCR-dependent NFATmediated T-cell activation with positron emission tomography in vivo. Neoplasia. 2001;3:480-488.

10. Green LA, Yap CS, Nguyen K, et al. Indirect monitoring of endogenous gene expression by positron emission tomography (PET) imaging of reporter gene expression in transgenic mice. Mol Imaging Biol. 2002;4:71-81.

11. Berger C, Flowers ME, Warren EH, Riddell SR. Analysis of transgene-specific immune responses that limit the in vivo persistence of adoptively transferred HSVTK-modified donor $\mathrm{T}$ cells after allogeneic hematopoietic cell transplantation. Blood. 2006;107:2294-2302.

12. Traversari C, Marktel S, Magnani Z, et al. The potential immunogenicity of the TK suicide gene does not prevent full clinical benefit associated with the use of TK-transduced donor lymphocytes in HSCT for hematologic malignancies. Blood. 2007;109:4708-4715.

13. Riddell SR, Elliott M, Lewinsohn DA, et al. T-cell mediated rejection of genemodified HIV-specific cytotoxic T lymphocytes in HIV-infected patients. Nat Med. 1996;2:216-223.

14. Likar Y, Dobrenkov K, Olszewska M, et al. PET imaging of HSV1-tk mutants with acquired specificity toward pyrimidine- and acycloguanosine-based radiotracers. Eur J Nucl Med Mol Imaging. 2009;36:1273-1282.

15. Che J, Doubrovin M, Serganova I, Ageyeva L, Zanzonico P, Blasberg R. hNISIRES-eGFP dual reporter gene imaging. Mol Imaging. 2005;4:128-136.

16. Moroz MA, Serganova I, Zanzonico P, et al. Imaging hNET reporter gene expression with ${ }^{124}$ I-MIBG. J Nucl Med. 2007;48:827-836.

17. Reubi JC, Kvols L, Krenning E, Lamberts SW. Distribution of somatostatin receptors in normal and tumor tissue. Metabolism. 1990;39(9, suppl 2):78-81.

18. MacLaren DC, Gambhir SS, Satyamurthy N, et al. Repetitive, non-invasive imaging of the dopamine D2 receptor as a reporter gene in living animals. Gene Ther. 1999;6:785-791.

19. Genove G, DeMarco U, Xu H, Goins WF, Ahrens ET. A new transgene reporter for in vivo magnetic resonance imaging. Nat Med. 2005;11:450-454.

20. Moore A, Josephson L, Bhorade RM, Basilion JP, Weissleder R. Human transferrin receptor gene as a marker gene for MR imaging. Radiology. 2001;221:244-250.

21. Ponomarev V, Doubrovin M, Shavrin A, et al. A human-derived reporter gene for noninvasive imaging in humans: mitochondrial thymidine kinase type 2 . $\mathrm{J} \mathrm{Nucl}$ Med. 2007;48:819-826.

22. Arner ES, Eriksson S. Mammalian deoxyribonucleoside kinases. Pharmacol Ther. 1995;67:155-186.

23. Radu CG, Shu CJ, Nair-Gill E, et al. Molecular imaging of lymphoid organs and immune activation by positron emission tomography with a new $\left[{ }^{18} \mathrm{~F}\right]$-labeled $2^{\prime}$ deoxycytidine analog. Nat Med. 2008;14:783-788.

24. Sabini E, Ort S, Monnerjahn C, Konrad M, Lavie A. Structure of human dCK suggests strategies to improve anticancer and antiviral therapy. Nat Struct Biol. 2003;10:513-519.

25. Iyidogan P, Lutz S. Systematic exploration of active site mutations on human deoxycytidine kinase substrate specificity. Biochemistry. 2008;47:47114720 .

26. Hazra S, Sabini E, Ort S, Konrad M, Lavie A. Extending thymidine kinase activity to the catalytic repertoire of human deoxycytidine kinase. Biochemistry. 2009;48: 1256-1263.

27. Ponomarev V, Doubrovin M, Serganova I, et al. Cytoplasmically retargeted HSV1-tk/GFP reporter gene mutants for optimization of noninvasive molecular-genetic imaging. Neoplasia. 2003;5:245-254.

28. Neschadim ALA, Sato T, Fowler D, Medin J. Novel lentiviral cell fate control gene therapy based on engineered variants of the human deoxycytidine kinase [abstract]. Mol Ther. 2008;16(suppl 1):S212.

29. Gong MC, Latouche JB, Krause A, Heston WD, Bander NH, Sadelain M. Cancer patient $\mathrm{T}$ cells genetically targeted to prostate-specific membrane antigen specifically lyse prostate cancer cells and release cytokines in response to prostate-specific membrane antigen. Neoplasia. 1999;1:123-127.

30. Rivière I, Sadelain M. Methods for the construction of retroviral vectors and the generation of high titer producers. Meth Mol Biol. 1997;7:59-78.

31. Gade TP, Hassen W, Santos E, et al. Targeted elimination of prostate cancer by genetically directed human T lymphocytes. Cancer Res. 2005;65:90809088 .

32. Tjuvajev JG, Stockhammer G, Desai R, et al. Imaging the expression of transfected genes in vivo. Cancer Res. 1995;55:6126-6132.

33. Alauddin MM, Conti PS. Synthesis and preliminary evaluation of 9-(4- $\left[{ }^{18} \mathrm{~F}\right]-$ fluoro-3-hydroxymethylbutyl)guanine $\left(\left[{ }^{18} \mathrm{~F}\right] \mathrm{FHBG}\right)$ : a new potential imaging agent for viral infection and gene therapy using PET. Nucl Med Biol. 1998;25:175-180.

34. Serganova I, Doubrovin M, Vider J, et al. Molecular imaging of temporal dynamics and spatial heterogeneity of hypoxia-inducible factor-1 signal transduction activity in tumors in living mice. Cancer Res. 2004;64:6101-6108. 
35. Gregor PD, Wolchok JD, Turaga V, et al. Induction of autoantibodies to syngeneic prostate-specific membrane antigen by xenogeneic vaccination. Int J Cancer. 2005; 116:415-421.

36. Stephan MT, Ponomarev V, Brentjens RJ, et al. T cell-encoded CD80 and 4-1BBL induce auto- and transcostimulation, resulting in potent tumor rejection. Nat Med. 2007;13:1440-1449.

37. Mwintshi K, Brennan DC. Prevention and management of cytomegalovirus infection in solid-organ transplantation. Expert Rev Anti Infect Ther. 2007;5:295-304.
38. Moomaw MD, Cornea P, Rathbun RC, Wendel KA. Review of antiviral therapy for herpes labialis, genital herpes and herpes zoster. Expert Rev Anti Infect Ther. 2003;1:283-295.

39. McSorley T, Ort S, Hazra S, Lavie A, Konrad M. Mimicking phosphorylation of Ser-74 on human deoxycytidine kinase selectively increases catalytic activity for dC and dC analogues. FEBS Lett. 2008;582:720-724.

40. Johansson M, Brismar S, Karlsson A. Human deoxycytidine kinase is located in the cell nucleus. Proc Natl Acad Sci USA. 1997;94:11941-11945. 